\title{
Relationship between Medical and Dental Health Expenditures of Industrial Workers
}

\author{
Masayuki Ueno ${ }^{1}$, Takashi Zaitsu², Akiko Oshiro ${ }^{3}$, Yoko Kawaguchi ${ }^{4}$
}

\begin{abstract}
Aim: There has been little research exploring the expenditures associated with medical and dental health services and their interrelationship. The purposes of this cross-sectional study were to describe features of annual health expenditure and to examine the relationship between medical and dental health expenditures.

Materials and methods: Data on health expenditure from a total of 9,149 haulage workers aged 18-75 years (7,343 men and 1,806 women), who belonged to a health insurance association, were drawn for the analysis using electric health insurance claims from January through December in 2015. In-patient and out-patient fees as well as the corresponding pharmaceutical fees reported in the health insurance claims were aggregated to derive total medical and dental health expenditures.

Results: The medical services utilization rate $(77.4 \%)$ was significantly higher than the dental services utilization rate $(42.0 \%)(p<0.001)$, and both medical and dental services utilization rates increased with age ( $p$ for trend $<0.001$ ). Per capita medical and dental health expenditures also significantly increased with age ( $p$ for trend $<0.001$ ). Per capita medical health expenditure in workers who used dental services was significantly higher than that in those who did not use dental services in persons in the 40 years and older age groups $(p<0.05)$.

Conclusion: The present findings indicate a positive association between medical and dental health expenditures. Therefore, an improvement in oral health through workplace preventive measures may bring decrease not only of dental health expenditure but also of medical and total health expenditures in the industry.

Keywords: Dental health, Health expenditure, Health insurance, Medical health, Workplace.

Journal of Oral Health and Community Dentistry (2019): 10.5005/jp-journals-10062-0046
\end{abstract}

\section{INTRODUCTION}

Japan has introduced a universal health insurance system for the entire population since 1961. Under this system, people can receive health services including prescription medicines and dental treatment at a relatively low rate, the same fee throughout the nation. There are many types of social health insurance plans covering different groups of people, which are managed by a society-managed health insurance, mutual aid associations, quasipublic national health insurance associations, citizens' health insurance, and late elders' health insurance. ${ }^{1}$

Regulatory measures through a fee schedule determined and standardized by the Japanese government have contained healthrelated costs compared with other countries. ${ }^{2}$ However, a steadily increasing national health expenditure in Japan has become a major concern. Japan spent 42.4 trillion yen (approximately 376 billion US dollars) on national health expenditure in the fiscal year 2015, an increase of $3.8 \%$ (1.6 trillion yen) more than in 2014 . $^{3}$ The reducing health expenditure, therefore, is a key issue to be tackled in Japan.

The same is true of many industrial companies. Because of limited financial resources, reducing healthcare-related expenses of workers is an important task for decision-makers in the industry. Correctly identifying and understanding the magnitude of health expenditure is indispensable to determining the amount of resources that can be allocated to other purposes.

It is well documented that oral health is closely related to general health. Dental disease, periodontal disease in particular, is strongly associated with systemic diseases such as diabetes and cardiovascular diseases. ${ }^{4,5}$ Since people at risk of dental disease are also considered to be at high risk of systematic disease, dental health expenditure may affect medical health expenditure. There are a few reports suggesting an association between medical and dental
${ }^{1}$ Department of Health Sciences, Saitama Prefectural University, Saitama, Japan; Department of Oral Health Promotion, Tokyo Medical and Dental University, Tokyo, Japan

${ }^{2,4}$ Department of Oral Health Promotion, Tokyo Medical and Dental University, Tokyo, Japan

${ }^{3}$ Dental Hospital, Tokyo Medical and Dental University, Tokyo, Japan

Corresponding Author: Masayuki Ueno, Department of Health Sciences, Saitama Prefectural University, Saitama, Japan; Department of Oral Health Promotion, Tokyo Medical and Dental University, Tokyo, Japan, Phone: +81-48-973-4752, e-mail: masayuki-ueno@spu.ac.jp

How to cite this article: Ueno M, Zaitsu T, et al. Relationship between Medical and Dental Health Expenditures of Industrial Workers. J Oral Health Comm Dent 2019;13(2):54-58.

Source of support: Research Fund of Clinical Study for Industrial Accident and Disease by the Japanese Ministry of Health, Labour and Welfare

Conflict of interest: None

health expenditures. ${ }^{6,7}$ As there has been little research exploring the expenditures associated with medical and dental health services and their interrelationship, currently available information is very limited. According to the 2015 annual report of the Japan health insurance association, dental health expenditure made up $10.7 \%$ of the total health expenditure. ${ }^{8}$ If a positive relationship between medical and dental health expenditures exists, the reduction of dental health expenditure could lead to the reduction of medical and total health expenditures. For an industrial company, such information would assist the management of healthcare-related expenses and the development of occupational health promotion policies.

The hypothesis to be tested in this study was that an increased dental health expenditure could also be associated with the inflated medical health expenditure. Therefore, the purposes of 
this cross-sectional study were to describe features of annual health expenditure and to examine the relationship between medical and dental health expenditures using the electronic health insurance data of haulage workers, the so-called transport industry workers, in a health insurance association.

\section{Materials and Methods \\ Data Collection}

The health expenditure data were drawn from a total of 9,149 haulage workers aged 18-75 years, who belonged to a health insurance association in a metropolitan area in Japan. Workers who were younger than 18 years or older than 75 years were excluded from the analysis. Information about health expenditure from January through December in 2015 was collected using electronic health insurance claims that the health insurance company owned. The data reflected expenditure for the medical and dental health services the workers received. Since all the available data of eligible workers were intended to be used in the study, no sample size determination was not made.

The demographic variables used were age and gender. As health expenditure related variables, in-patient and out-patient fees as well as the corresponding pharmaceutical fees reported in the health insurance claims were aggregated to derive the total medical and dental health expenditures for the 12-month period. Medical health expenditure was matched and linked with dental health expenditure using an individually assigned identification number. Private patients' medical or dental treatment fees such as orthodontic treatments and any prosthetic appliance such as implants were excluded from the analysis. Currency conversion was done at the rate of 1 US dollar to 122.05 yen, the average rate in 2015.

The consent of usage of the health insurance claims data of workers was obtained from the health insurance association and companies belonging to the association. Ethical approval was granted by the Tokyo Medical and Dental University Ethics Committee (Approval number: D2016-054).

\section{Statistical Analysis}

Demographic characteristics of the study subjects were based on age group (18-39 years, 40-59 years, and 60-75 years) and gender. The proportions of subjects who used medical or dental services and mean and standard error (SE) of per capita medical or dental health expenditure by age group were calculated. Medical and dental health expenditures were adjusted for age and gender using an analysis of covariance. Linear trends were tested using the Mantel-Haenszel's Chi-square statistics for medical or dental utilization rate by age group and the Jonckheere-Terpstra test for mean medical or dental health expenditure by age group. Significant differences in the mean medical health expenditure between subjects who used or did not use dental services were assessed by the independent $t$ test. Multiple comparisons for the mean medical health expenditure among the tertile of dental health expenditure or number of days of dental visits were analyzed using a Bonferroni method. All statistical procedures were implemented with the IBM ${ }^{\circledR}$ SPSS $^{\circledast} 23.0$ (IBM Japan Corp., Tokyo, Japan).

\section{Results}

\section{Demographic Characteristics of Subjects}

Table 1 shows the basic characteristics of the subjects. There were 9,149 subjects: 7,343 men and 1,806 women. The overall mean age was 43.94 (0.14 SE) years; men 44.83 (0.15 SE) years and women 40.33 ( $0.29 \mathrm{SE}$ ) years. Age group $18-39$ years was $38.3 \%$ of the total, $40-59$ years was $46.1 \%$, and $60-75$ years was $15.6 \%$. Those in men were $35.8 \%, 46.6 \%$, and $17.6 \%$ and in women were $48.6 \%, 43.9 \%$, and $17.5 \%$, respectively.

\section{Utilization of Medical and Dental Services}

Overall medical and dental services utilization rates were $77.4 \%$ and $42.0 \%$; rate was significantly higher for medical services $(p<0.001)$ (Table 2). The medical services utilization rates by age group were $73.0 \%$ in the $18-39$ years age group, $76.9 \%$ in the $40-59$ years, and $89.4 \%$ in the $60-75$ years age groups. Dental services utilization rates by age group were $36.5 \%$ in $18-39$ years, $43.3 \%$ in $40-59$ years, and $51.5 \%$ in $60-75$ years age groups. The medical services utilization rate was nearly double that of the dental services rate and significantly higher in every age group ( $p<0.001$ for all age groups). For both medical and dental services utilization rates, there was a statistically significant increasing trend with age ( $p$ for trend $<0.001$ and $p$ for trend $<0.001$ ).

Overall medical services utilization rates in subjects who used or did not use dental services were $85.2 \%$ and $71.7 \%$, respectively. The medical services utilization rate was significantly higher in subjects who used dental services than among those who did not $(p<0.001)$ (Table 3). The comparison by age group also showed that medical services utilization rates were significantly higher in subjects who used dental services than among those who did not ( $p<0.001$ for all age groups). For both dental services users and nondental services

Table 1: Subjects by age group and gender

\begin{tabular}{lccr}
\hline $\begin{array}{l}\text { Age group, } \\
\text { years }\end{array}$ & Men, \%(n) & Women, \% $(n)$ & \multicolumn{1}{c}{ Total, \% $(n)$} \\
\hline $18-39$ & $35.8(2,627)$ & $48.6(878)$ & $38.3(3,505)$ \\
$40-59$ & $46.6(3,422)$ & $43.9(792)$ & $46.1(4,214)$ \\
$60-75$ & $17.6(1,294)$ & $17.5(136)$ & $15.6(1,430)$ \\
All subjects & $100.0(7,343)$ & $100.0(1,806)$ & $100.0(9,149)$ \\
\hline
\end{tabular}

Table 2: Proportion of subjects who used medical and dental services

\begin{tabular}{llll}
\hline \multirow{2}{*}{$\begin{array}{l}\text { Age group, } \\
\text { years }\end{array}$} & \multicolumn{2}{c}{$\begin{array}{c}\text { Health services utilization rate, } \\
\%(n)\end{array}$} & $\begin{array}{l}\text { p value for } \\
\text { proportional } \\
\text { difference }\end{array}$ \\
\cline { 2 - 4 } & Medical & Dental & $<0.001$ \\
$18-39$ & $73.0(2,560)$ & $36.5(1,278)$ & $<0.001$ \\
$40-59$ & $76.9(3,239)$ & $43.3(1,825)$ & $<0.001$ \\
$60-75$ & $89.4(1,278)$ & $51.5(737)$ & $<0.001$ \\
All subjects & $77.4(7,077)$ & $42.0(3,840)$ & \\
$p$ for trend & $<0.001$ & $<0.001$ & \\
\hline
\end{tabular}

Table 3: Proportion of subjects who used medical services by dental services use

\begin{tabular}{llll}
\hline & \multicolumn{2}{c}{$\begin{array}{c}\text { Medical services utilization rate, } \\
\text { \% }(n)\end{array}$} & $\begin{array}{l}\text { p value for } \\
\text { proportional } \\
\text { difference }\end{array}$ \\
\cline { 2 - 3 } $\begin{array}{l}\text { Age group, } \\
\text { years }\end{array}$ & $\begin{array}{l}\text { Dental services } \\
\text { users }\end{array}$ & $\begin{array}{l}\text { Nondental } \\
\text { services users }\end{array}$ & $<0.001$ \\
\hline $18-39$ & $81.4(1,040)$ & $68.3(1,520)$ & $<0.001$ \\
$40-59$ & $84.2(1,536)$ & $71.3(1,703)$ & $<0.001$ \\
$60-75$ & $94.2(694)$ & $84.3(584)$ & $<0.001$ \\
All subjects & $85.2(3,270)$ & $71.7(3,807)$ & \\
$p$ for trend & $<0.001$ & $<0.001$ & \\
\hline
\end{tabular}


users, a statistically significant increasing trend of utilization rate with age was shown ( $p$ for trend $<0.001$ and $p$ for trend $<0.001$ ).

\section{Per Capita Medical and Dental Health Expenditures}

Table 4 presents the per capita medical and dental health expenditures by age group. Overall, these expenditures in all subjects were $\$ 1,052.61$ and $\$ 161.74$ per capita, respectively. Per capita medical health expenditures by age group were $\$ 467.63$ in the $18-39$ years group, $\$ 1,060.04$ in the $40-59$ years group, and $\$ 2,464.56$ in the $60-75$ years group. Per capita dental health expenditures by age group were \$123.71, \$172.31, and $\$ 223.79$, respectively. Both per capita medical and dental health expenditures significantly increased with age ( $p$ for trend $<0.001$ and $p$ for trend $<0.001$ ).

Overall per capita medical and dental health expenditures in subjects who used health services were $\$ 1,360.80$ and $\$ 385.35$, respectively. Per capita medical health expenditures by age group were $\$ 647.41$ in subjects $18-39$ years old, $\$ 1,377.84$ in $40-59$ year olds, and $\$ 2,746.63$ in $60-75$ year olds. Per capita dental health expenditures by age group were $\$ 344.56, \$ 397.50$, and $\$ 426.00$, respectively, in the same age groups. Both per capita medical and dental health expenditures also had significant increases with age ( $p$ for trend $<0.001$ and $p$ for trend $<0.001$ ).

\section{Per Capita Medical Health Expenditure According to Dental Services Utilization}

Overall per capita medical health expenditure in subjects who used dental services was $\$ 1,219.12$, which was significantly higher than $\$ 932.18$ in those who did not $(p<0.001)$. Per capita medical health expenditures in subjects who used dental services by age group were $\$ 571.75, \$ 1,217.83$, and $\$ 2,850.28$ in the three age groups and in those who did not use dental services were $\$ 419.65$, $\$ 937.15$, and $\$ 2,024.62$, respectively (Table 5). Per capita medical health expenditures in both dental services users and nondental services users increased significantly with age ( $p$ for trend $<0.001$ and $p$ for trend $<0.001$ ). Significant mean differences in the per capita medical health expenditure by the use of dental services were found in the 40-59 and 60-75 years age groups ( $p=0.036$ and $p=0.010$ ).

Among subjects who used dental services, per capita medical health expenditure by the tertile of per capita dental health expenditure $(<\$ 170.27, \$ 170.27-\$ 400.99$ and $>\$ 400.99)$ or number of days of dental visits (1-3 days, 4-7 days, and $>7$ days) were $\$ 1,414.98(n=1,280), \$ 1,417.93(n=1,281)$, and $\$ 1,124.57(n=1,279)$ or $\$ 1,381.27(n=1,454), \$ 1,307.70(n=1,113)$, and $\$ 1,258.48(n=1,273)$, respectively (Table 6 ). No significant differences in per capita medical health expenditure were detected by the tertile of dental health expenditure or number of days of dental visits.

\section{Discussion}

This cross-sectional study used the data drawn from health insurance claims of national health insurance; therefore, patients' private fees for care were not included in the analysis. Japanese national health insurance covers both medical and dental care, and dental care covers most restorative and surgical treatments such as fillings, endodontics, crowns, bridges, dentures, and extractions. According to the Survey on Economic Conditions in Health Care in $2015,{ }^{9}$ the proportion of dental expenses provided by the national health insurance is about $85.8 \%$ of the total dental health expenditure. The proportion of expenses for medical healthcare borne by private fees only was $1.3 \%$ in $2015 .{ }^{3}$ Further, the mean per capita medical and dental health expenditures in the sample were close to those reported in the national health expenditure report in $2015 .^{3}$ These statistics suggest that our data are a good estimate of Japanese health expenditure.

The findings show a positive relationship between medical and dental health expenditures. Both medical services use and medical health expenditure increased with age. The same positive gradient in dental services use and dental health expenditure by

Table 4: Per capita medical and dental health expenditures (US \$) in all subjects and those who used health services, $\operatorname{mean}^{\dagger}(\mathrm{SE})$

\begin{tabular}{llllll}
\hline & \multicolumn{4}{c}{ Health expenditure, mean (SE) } \\
\cline { 2 - 3 } \cline { 6 - 7 } Age group, years & Medical & \multicolumn{2}{c}{ Dental } & & \multicolumn{2}{c}{ Health services users } \\
\cline { 2 - 3 } \cline { 6 - 7 } & $467.63(65.11)$ & $123.71(6.48)$ & & $647.41(85.95)$ & $344.56(14.43)$ \\
$40-59$ & $1,060.04(59.19)$ & $172.31(5.89)$ & & $1,377.84(75.99)$ & $397.50(12.01)$ \\
$60-75$ & $2,464.56(102.12)$ & $223.79(10.16)$ & & $2,746.63(121.88)$ & $426.00(19.07)$ \\
All subjects & $1,052.61(40.14)$ & $161.74(3.99)$ & & $1,360.80(51.34)$ & $385.35(8.27)$ \\
$p$ for trend & $<0.001$ & $<0.001$ & & $<0.001$ & $<0.001$ \\
\hline
\end{tabular}

†Adjusted for age and gender

Table 5: Per capita medical health expenditure (US \$) in subjects who used and who did not use dental services, $\operatorname{mean}^{\dagger}(\mathrm{SE})$

\begin{tabular}{lllc}
\hline & \multicolumn{2}{c}{ Medical health expenditure, mean (SE) } & $\begin{array}{c}\text { p value for mean } \\
\text { difference }\end{array}$ \\
\cline { 2 - 3 } Age group, years & Dental services users & Nondental services users & 0.081 \\
\hline $18-39$ & $571.75(69.33)$ & $419.65(52.47)$ & 0.036 \\
$40-59$ & $1,217.83(89.44)$ & $937.15(78.14)$ & 0.010 \\
$60-75$ & $2,850.28(222.18)$ & $2,024.62(229.13)$ & $<0.001$ \\
All subjects & $1,219.12(62.32)$ & $932.18(52.91)$ & \\
$p$ for trend & $<0.001$ & $<0.001$ & \\
\hline
\end{tabular}

†Adjusted for age and gender 
Relationship between Medical and Dental Health Expenditures of Industrial Workers

Table 6: Per capita medical health expenditure (US \$) by the tertile of dental health expenditure and number of days of dental visits, mean ${ }^{\dagger}$ (SE)

\begin{tabular}{|c|c|c|c|c|c|}
\hline \multicolumn{3}{|c|}{ Tertile of dental health expenditure } & \multicolumn{3}{|c|}{ Tertile of number of days of dental visits } \\
\hline$<\$ 170.27$ & $\$ 170.27-\$ 400.99$ & $>\$ 400.99$ & $1-3$ days & 4-7 days & $>7$ days \\
\hline $1,414.98(124.70)$ & $1,417.93(124.23)$ & $1,124.57(124.76)$ & $1,381.27(116.99)$ & $1,307.70(133.39)$ & $1,258.48(125.34)$ \\
\hline
\end{tabular}

${ }^{\dagger}$ Adjusted for age and gender

age was also observed. Previous studies found a similar association among dental services use, dental health expenditure, and age. ${ }^{10-13}$ A Japanese web-based study showed that the frequency of dental visits and dental treatment increased with age. ${ }^{13}$ Middleaged or older adults were reported to have greater dental health expenditure than younger adults. ${ }^{11,12}$ Australian people who visited a dental provider more than once a year on average had a higher dental health expenditure than those who visited less often, and a poorer oral health status was related with higher dental health expenditure. ${ }^{10}$ These findings imply that the need for dental services might increase due to poorer oral health conditions with age, leading to increased dental health expenditure. Having dental health insurance has also been suggested to be associated with dental visits and dental services provision and thus dental health expenditure, in other countries, ${ }^{12,14}$ but the effect of health insurance could be minimal because of the universal health insurance system in Japan.

Medical health expenditure in subjects who did not use dental services was lower than those who used the services and the amount of dental health expenditures or the frequency of dental services use did not influence the medical health expenditure. The difference of medical health expenditure by dental services use expanded in the middle and old age groups. It is well known that oral health is closely linked with general health. ${ }^{15-19}$ People who have good oral health usually have good general health, and the subjects in our study who did not use dental services were also less likely to use medical services. A significant relationship between the subjective assessment of oral health and medical health expenditure has been reported in a previous study. Since people with good subjective oral health were considered to have good general health, they were less likely to use medical services and had lower medical expenses as a consequence. ${ }^{20}$

Periodontal disease has also been ascribed to the increase in medical health expenditure. People with severe periodontitis incurred higher medical health expense, probably due to the deterioration of general health caused by periodontal diseases. ${ }^{18,21}$ The difference in medical health expenditure by dental services use observed in subjects aged 40 years or older in this study might partially be due to the increased prevalence of periodontitis. The number of teeth present was another important factor associated with medical health expenditure; people with fewer teeth incurred greater medical health expenditure. ${ }^{22,23}$ However, the absence of information about oral health status makes it difficult to determine whether subjects used dental services less because they had no dental problems or, despite having poor dental condition, they had other reasons for avoiding dental care.

This study is subject to a couple of other limitations. First, since details of the dental care provided were not available, it was not possible to identify the type of dental services responsible for the expenditure. Second, the study used only the data of insured workers in a health insurance association. Inclusion of their family members' data would have given a more complete picture.
Within such limitations, the present findings suggest that oral health is an important contributor to health expenditure. Although dental health expenditure is a small proportion of total health expenditure, a reduction of dental health expenditure can contribute to reducing the economic burden of healthcare. Further, an improvement in oral health might bring benefits not only in terms of reduced health expenditure but also increase labor productivity by virtue of better general health.

A workplace is considered a suitable place for conducting oral health promotion activities, because many workers spend much time there. Several studies have reported that workplace oral health promotion activities contribute to reducing healthcare expenses and are cost-beneficial for employers. ${ }^{6,24,25}$ Annual oral examinations and tooth brushing instruction in the workplace have been demonstrated to be beneficial for preventing dental diseases. ${ }^{26}$ Prevention of dental diseases, periodontal disease in particular, requires coordinated efforts from various sections. ${ }^{27}$ Most Japanese adults have varying levels of periodontal disease, even though it is preventable. Unlike medical examinations, dental examinations are rarely undertaken in the workplace. Dental examination with accompanying health education to provide basic information about periodontal disease and risk factors, including smoking, should be offered to all workers as a part of the health examination.

\section{Conclusion}

Health insurance claims can provide an accurate and reliable source of health expenditure information. Additional analysis of health expenditure with linked information about clinical oral status and detailed service type needs to be conducted to confirm current findings on the positive relationship between medical and dental health expenditures and to reveal dental conditions or treatment procedures associated with health expenditure of industrial workers.

\section{Acknowledgment}

This study was financially supported by the "Research Fund of Clinical Study for Industrial Accident and Disease" (Grant No. 14020101-01 and 170501-01) from the Japanese Ministry of Health, Labour and Welfare. The authors are deeply grateful to staff members of ai-Health Corp for their generous cooperation and support.

\section{References}

1. Ikegami N, Yoo BK, et al. Japanese universal health coverage: evolution, achievements, and challenges. Lancet 2011;378(9796): 1106-1115. DOI: 10.1016/S0140-6736(11)60828-3.

2. OECD. Health expenditure and financing 2017. Available from: URL: http://stats.oecd.org/Index.aspx?DataSetCode=SHA 2017.

3. Ministry of Health, Labour and Welfare. National health expenditures in fiscal year 2015. Available from: http://www.mhlw.go.jp/toukei/ saikin/hw/k-iryohi/15/dl/kekka.pdf. 
4. Ueno $M$, Takeuchi S, et al. Association between diabetes mellitus and oral health status in Japanese adults. Int J Oral Sci 2010;2(2):82-89. DOI: $10.4248 /$ IJOS10025.

5. Linden GJ, Herzberg MC, et al. Periodontitis and systemic diseases: a record of discussions of working group 4 of the joint EFP/AAP workshop on periodontitis and systemic diseases. J Periodontol 2013;84:S20-S23. DOI: 10.1902/jop.2013.1340020.

6. Ichihashi T, Muto T. Effectiveness of worksite dental health promotion activities in terms of dental and medical expenses and number of visits for treatment. J Dent Health 2001;51:168-175.

7. Takeuchi K, Sato Y, et al. Associations of oral health status and dental health service utilization with dental and medical expenditures. J Dent Health 2017;67:160-171.

8. Japan Health Insurance Association. Annual report in fiscal year 2015. Available from: https://www.kyoukaikenpo.or.jp/g7/cat740/sb7200/ sbb7200/270407.

9. Ministry of Health, Labour, and Welfare. Survey on Economic Conditions in Health Care in 2015. Available from: http://www. mhlw.go.jp/bunya/iryouhoken/database/zenpan/jittaityousa/ dl/20_houkoku_iryoukikan.pdf.

10. Teusner DN, Brennan DS, et al. Individual dental expenditure by Australian adults. Aust Dent J 2013;58(4):498-506. DOI: 10.1111/ adj.12108.

11. Sivaneswaran $\mathrm{S}$, Taylor $\mathrm{R}$, et al. Cost of dental services provided in private general practice for an insured population in New South Wales, Australia. Comm Dent Health 2000;17(4):246-253.

12. Brennan DS, Spencer AJ, et al. Insurance status and provision of dental services in Australian private general practice. Comm Dent Oral Epidemiol 1997;25(6):423-428. DOI: 10.1111/j.1600-0528.1997. tb01733.x.

13. Ando $Y$, Ishida $T$, et al. The status of routine dental visits by web-based survey in Japan. J Dent Health 2012;62(1):41-52.

14. Elstad JI. Dental care coverage and income-related inequalities in foregone dental care in Europe during the great recession. Comm Dent Oral Epidemiol 2017;45(4):296-302. DOI: 10.1111/cdoe. 12288.

15. Azarpazhooh A, Leake JL. Systematic review of the association between respiratory diseases and oral health. J Periodontol 2006;77(9):1465-1482. DOI: 10.1902/jop.2006.060010.
16. Östberg AL, Bengtsson C, et al. Oral health and obesity indicators. BMC Oral Health 2012;12:50. DOI: 10.1186/1472-6831-12-50.

17. Ueno $M$, Izumi $Y$, et al. Prediagnostic plasma antibody levels to periodontopathic bacteria and risk of coronary heart disease. Int Heart J 2012;53(4):209-214. DOI: 10.1536/ihj.53.209.

18. Ide R, Hoshuyama $T$, et al. The effect of periodontal disease on medical and dental costs in a middle-aged Japanese population: a longitudinal worksite study. J Periodontol 2007;78(11):2120-2126. DOI: 10.1902/jop.2007.070193.

19. Ericsson JS, Ostberg AL, et al. Oral health-related perceptions, attitudes, and behavior in relation to oral hygiene conditions in an adolescent population. Eur J Oral Sci 2012;120(4):335-341. DOI: 10.1111/j.1600-0722.2012.00970.x.

20. Harada E, Moriya S, et al. Relationship between subjective assessment of oral health and medical expenses in community-dwelling elderly persons. Gerodontology 2012;29(2):e246-e252. DOI: 10.1111/j.17412358.2011.00459.x.

21. Sato $M$, Iwasaki $M$, et al. Association between periodontitis and medical expenditure in older adults: a 33-month follow-up study. Geriatr Gerontol Int 2016;16(7):856-864. DOI: 10.1111/ggi.12569.

22. Kanda $\mathrm{M}$, Ueda $\mathrm{H}$, et al. A three-year follow-up study of the relationship among the numbers of present teeth, the loss of teeth and medical expenditure in the elderly. Jpn J Gerodontol 2008;23:132-139.

23. Tsuneishi $M$, Yamamoto $T$, et al. Association between number of teeth and medical and dental care expenditures - Analysis using the receipt and health checkup information database in Japan. Jpn J Dent Prac Admin 2016;51:136-142.

24. Ichihashi T, Muto T, et al. Cost-benefit analysis of a worksite oral-health promotion program. Ind Health 2007;45(1):32-36. DOI: 10.2486/ indhealth.45.32.

25. Ide R, Mizoue T, et al. Evaluation of oral health promotion in the workplace: the effects on dental care costs and frequency of dental visits. Comm Dent Oral Epidemiol 2001;29(3):213-219. DOI: 10.1034/j.1600-0528.2001.290307.x.

26. Zaitsu T, Kanazawa T, et al. Relationships between occupational and behavioral parameters and oral health status. Ind Health 2017;55: 381-390. DOI: 10.2486/indhealth.2017-0011.

27. Nair AR, Prashant GM, et al. Dental education: challenges and changes. J Oral Health Comm Dent 2017;11(2):34-37. 